\title{
Therapeutic effect of human umbilical cord blood cells on diebetic mice
}

\author{
Fang-ting Zhang ${ }^{1}$, Hui-juan Wan ${ }^{1}$, Jing Ye $^{1}$, Jie Yu ${ }^{1}$, Xia Long ${ }^{1}$, Mei-jun Yin ${ }^{1}$, Jia-zhi Fang ${ }^{1}$ \\ ${ }^{1}$ Central Laboratory, Peking University Shenzhen Hospital, China
}

Human adult stem cell research has been paid more attention because of the compelling ethical and legal issues surrounding human embryonic stem cells. Mononuclear cells from human umbilical cord blood (MNCs) have been showed to have plasticity. In our study, human umbilical cord blood MNCs could survival in diabetic mice without using immuno-suppressive drugs and could decreased serum glucose levels at definite time period. To observe the therapeutic effect on diabetic mice of mononuclear cell from human umbilical cord blood, experimental diabetes animal model was induced in $8 \mathrm{w}$ kun-ming mice by a single intraperitioneal injection of streptozotocin freshly dissolved in 0.1M of citrate buffer, pH4.5; serum glucose level were observed to monitoring effect on diabetic model. Freshly isolated human umbilical cord blood cells were labeled using PKH26, then transplanted at $2 \times 10^{6} / \mathrm{ml}$ via tail vein into the model animal. glucose and insulin level in serum was determined at different time point, namely, after transplantation and distribution of transplanted cells in vivo were also examined by flow cytometer and fluorescence microscope. After 4d of cell transplantation, the serum glucose level significantly decreased and the serum insulin level increased in comparison with pre-transplantation. After 14d, the serum glucose level was recovered to the pre-transplantation level but the serum insulin level decreased in comparison with $4 \mathrm{~d}$ and became no significant difference when compared with no-transplantation STZ treated group. PKH26 positive cells were found in panaceas, spleen, liver and bone marrow of transplanted group mice at $4 \mathrm{~d}$, but not in above-mentioned position at $14 \mathrm{~d}$ by flow cytometer. The result of fluorescence microscope on tissue section were also supported this consequence.

Keywords: human umbilical cord blood, mononuclear cells, diabetic mice

Cell Research (2008) 18:s48. doi: 10.1038/cr.2008.138; published online 4 August 2008

Correspondence: Fang-ting Zhang

E-mail: fangtingzhang@126.com 